\title{
Laboratory diagnostic approaches in metabolic disorders
}

\author{
Ruben Bonilla Guerrero ${ }^{1}$, Denise Salazar ${ }^{2}$, Pranoot Tanpaiboon $^{2,3}$ \\ ${ }^{1}$ Formerly Quest Diagnostics, Inc., Ruben Bonilla Guerrero, Rancho Santa Margarita, CA, USA; ${ }^{2}$ Quest Diagnostics, Inc., Denise Salazar and \\ Pranoot Tanpaiboon, San Juan Capistrano, CA, USA; ${ }^{3}$ Genetics and Metabolism, Children's National Rare Disease Institute, Washington, DC, USA \\ Contributions: (I) Conception and design: All authors; (II) Administrative support: R Bonilla Guerrero; (III) Provision of study materials or patients: \\ All authors; (IV) Collection and assembly of data: All authors; (V) Data analysis and interpretation: None; (VI) Manuscript writing: All authors; \\ (VII) Final approval of manuscript: All authors. \\ Correspondence to: Ruben Bonilla Guerrero. Formerly Quest Diagnostics, Inc., Ruben Bonilla Guerrero, 508 Sable, Rancho Santa Margarita, CA \\ 92688, USA. Email: bonilla.ruben@ymail.com.
}

\begin{abstract}
The diagnosis of inborn errors of metabolism (IEM) takes many forms. Due to the implementation and advances in newborn screening (NBS), the diagnosis of many IEM has become relatively easy utilizing laboratory biomarkers. For the majority of IEM, early diagnosis prevents the onset of severe clinical symptoms, thus reducing morbidity and mortality. However, due to molecular, biochemical, and clinical variability of IEM, not all disorders included in NBS programs will be detected and diagnosed by screening alone. This article provides a general overview and simplified guidelines for the diagnosis of IEM in patients with and without an acute metabolic decompensation, with early or late onset of clinical symptoms. The proper use of routine laboratory results in the initial patient assessment is also discussed, which can help guide efficient ordering of specialized laboratory tests to confirm a potential diagnosis and initiate treatment as soon as possible.
\end{abstract}

Keywords: Metabolic; inborn; disorder; diagnosis; laboratory

Submitted Oct 01, 2018. Accepted for publication Oct 17, 2018.

doi: $10.21037 /$ atm.2018.11.05

View this article at: http://dx.doi.org/10.21037/atm.2018.11.05

\section{Introduction}

The term "inborn errors of metabolism" (IEM) was first described by Sir Archibald Garrod in 1908 (1) to describe those diseases caused by a block in a metabolic pathway due to the deficient activity of a specific enzyme. In recent years, advancement in technologies such as tandem mass spectrometry (MS/MS) and next-generation sequencing (NGS) employing a massive parallel sequencing strategy, have profoundly expanded our knowledge of IEM and metabolic disorders in general. These new technologies have also allowed for the expansion and improvement of newborn screening (NBS) on a global scale. In the United States (US), the Secretary's Advisory Committee on Heritable Disorders in Newborns and Children (ACHDNC) (part of the US Department of Health and Human Services) recommends that all newborns be screened for 34 core disorders as well as 26 secondary disorders. Approximately $80 \%$ of the disorders tested in NBS are IEM (2). The expansion of newborn metabolic screening helps identify pre-/asymptomatic newborns, leading to early treatment. However, there is still the need to identify individuals whose defect was missed by NBS, or who were born before widespread screening was available.

\section{Background}

IEM can be classified into two broad categories: those which affect energy production, and those which affect the synthesis or degradation of specific molecules or compounds. Although carbohydrates, fats, and proteins are all used as energy sources, the degree to which a specific fuel is utilized depends on the organ or tissue type. A disruption to a metabolic process that affects one type of fuel leads to the increased utilization of an alternate fuel 
type; this compensatory shift in energy production can lead to a pattern of abnormal metabolites observed in patients with these disorders, which is key to making the diagnosis. Due to the complex regulation of metabolic processes throughout the body, the accumulation of a substrate from a blocked metabolic step will engage alternate metabolic pathways that are otherwise minimally utilized under normal circumstances, leading to an increase in the production and accumulation of potentially harmful intermediate metabolites in patients with IEMs. These intermediates may also interfere with normal metabolism through activation or inhibition of enzymatic processes, or through competitive action, leading to additional clinical symptoms and pathognomonic patterns of elevated analytes. Most organic acidurias, amino acidopathies, peroxisomal disorders, lysosomal storage disorders (LSD), glycogen storage disorders (GSD), and mitochondrial fatty acid oxidation disorders are examples of defects in degradative pathways, in which specific enzymes break down substrates (glycogen, organic acids, amino acids, or fatty acids) to produce energy or to generate basic building blocks utilized in subsequent synthetic processes (i.e., creatine synthesis). In contrast, the porphyrias, cerebral creatine deficiencies, and congenital disorders of glycosylation are examples of defects in synthetic pathways, which impact the production of heme, creatine, and glycoproteins respectively. Transporters and channel proteins which mobilize substrate fall into both categories (3-6).

\section{General approach to diagnosing IEM}

Patients with inherited metabolic disorders can create a familiar diagnostic dilemma in clinical practice. Even when there is a strong suspicion of an underlying metabolic defect, the sheer number of disorders and the variability in clinical features can render diagnostic test selection difficult. Clinical phenotypes of IEM are broad and often non-specific, mimicking more common conditions, and the onset of symptoms can occur at any age from fetus to adult. Although the classical presentation of most IEM involves an acutely ill newborn or young infant, this scenario is encountered less frequently now that advances in tandem mass-spectrometry-based NBS have decreased the number of acute episodes and consequent sequelae for many of the conditions identified by the screening test. However, because patients with milder mutations and subtle biochemical phenotypes may be missed by the cut-off values used to determine positive screening results, a normal newborn screen should not exclude these disorders from the differential diagnosis in a patient whose clinical presentation is suggestive of an inherited metabolic defect. Both clinical and biochemical phenotypes of IEM in children have been studied for decades; conversely, late onset or adult onset variants were largely unrecognized. In recent years, adult onset forms of many inherited metabolic disorders have been increasingly identified as true mild phenotypes, where symptoms in childhood were not severe enough to merit investigation. In these late onset variants of metabolic disorders, some not presenting until well into adulthood, the clinical features may be significantly different from the classical features associated with the underlying disorder, which, along with the patient's age, could lead to a significant delay in diagnosis.

Appropriate and prudent test selection must be driven by a combination of the patient's clinical presentation and the results of routine first tier laboratory tests, which can help guide more specific testing. Depending on the affected metabolic pathway, the clinical presentation may include hallmark manifestations characteristic of and/or common to a group of disorders; however, such features are not typically unique to a single disorder. When evaluating a patient for a possible IEM, routine laboratory tests can identify underlying patterns suspicious for a metabolic defect. Common findings include hypoketotic hypoglycemia, lactic acidosis, metabolic acidosis, ketosis, hyperammonemia, or metabolic acidosis in combination with hyperammonemia. Evaluating these blood and urine test results in conjunction with the clinical presentation can narrow the focus toward a particular subset of metabolic disorders. Among the clues that should lead clinicians to suspect an IEM are scenarios such as a critically ill neonate with a history of deterioration following an uncomplicated pregnancy, episodes of illness or fluctuating symptoms of lethargy or other neurological symptoms precipitated by intercurrent illness or stress, multisystem involvement, failure to thrive, developmental delay, progressive neurological signs or bizarre neurological symptoms with or without psychological problems in patients in whom the usual etiologies have been excluded, particularly in adults (7). Although biochemical genetic and molecular genetic tests are required to confirm a diagnosis, basic laboratory tests are still important and often provide the first clues to a possible underlying IEM. Medical and family history, clinical symptoms, and basic labs are usually adequate to categorize an IEM and provide initial treatment before the results of more specific testing are available, especially in an emergency situation. 
Table 1 Routine laboratory tests that should be always ordered when an IEM is suspected

Test
Complete blood count with differential
Liver function tests
Renal function tests
*Ammonia
Blood gases
Anion gap
Glucose
Lactate
Urine and blood ketones

This table represents the most useful routine laboratory tests when suspecting an IEM in a patient with a metabolic stress state. Results of these tests may indicate the underlying pathophysiology and narrow the focus of additional testing to identify a metabolic disorder or category of disorders. *, red blood cells can produce and release ammonia into standing blood specimen. In addition, enzymes in blood can deaminate amino acids causing elevation of ammonia. Therefore, measurement of ammonia can be challenging. A free-flowing blood sample should be collected into preferable pre-chilled tubes. The sample should be transported on ice to the laboratory and separated as soon as possible, preferably within 15 minutes. Specimen should be analyzed as soon as possible. IEM, inborn errors of metabolism.

The first step in helping to select the appropriate laboratory investigation to rule out an IEM is to determine whether the condition is likely due to defects in small molecule metabolism (such as disorders of amino acids, organic acids, purines and pyrimidines, the urea cycle, mitochondrial energy metabolism) or defects of organelle metabolism (such as lysosomes or peroxisomes) (8-10). Patients with small molecule disorders usually present with acute illness requiring emergency intervention. Patients with disorders of organelle metabolism commonly present with neurological and neuromuscular manifestations, organomegaly, hepatic dysfunction, with or without dysmorphism. However, it is important to keep in mind that in some instances, disorders affecting organelle function may also present with metabolic crises including hypoglycemia and/or metabolic acidosis requiring emergency intervention. Additionally, it is important to recognize that some IEM may present without a life threatening metabolic crisis, but rather with unusual symptoms like skin blistering after sunlight exposure and/ or neurological manifestations (i.e., cryptogenic abdominal pain, paresthesia, or psychotic episodes) as is the case for most porphyrias; exceptions are congenital erythropoietic porphyria (CEP) which can produce severe liver disease and acute intermittent porphyria (AIP) which can produce severe lactic acidosis (4).

\section{Small molecule metabolism defects}

Patients with defects of small molecule metabolism may first present with acute illness. Basic laboratory tests should be performed in every child with an acute illness in whom an underlying metabolic disorder is a possibility. The following tests are examples of basic but nevertheless critical investigations to be considered when evaluating a patient for a potential IEM, as results can be directly affected by these disorders. Results of basic testing can provide direction into the underlying etiology and help in the selection of further more specific tests (Table 1).

\section{Acid-base status to determine the anion gap}

Metabolic acidosis is a disturbance in the body's acid-base balance through the loss of bicarbonate, reduced renal excretion, or increased production of acids. Determining the acid-base status is important in the assessment of a patient with a potential inherited metabolic defect, because high anion gap metabolic acidosis is usually caused by the accumulation of organic acids including lactic acid, ketone bodies, or unusual acids and their derivatives. In contrast, diarrhea and renal tubular acidosis are the main causes of metabolic acidosis with a normal anion gap (11). If acidosis is present, it should be evaluated in conjunction with other metabolic states such as hypo- and hyper-glycemia, ketosis, hyperlactatemia, and hyperammonemia. The majority of IEM that present with overwhelming metabolic acidosis and ketosis are organic acidemias (i.e., methylmalonic acidemia, propionic acidemia, isovaleric acidemia). On the other hand, metabolic acidosis with hypoglycemia and no ketosis can be the only finding in an underlying mitochondrial fatty acid oxidation defect, where rescue of hypoglycemia is impaired by the inability to produce energy from fatty acid metabolism and an increase in the production of non-physiological dicarboxylic acid intermediates (12). Metabolic acidosis and hyperlactatemia in the absence of elevated organic acids other than lactic acid and pyruvic acid can be found in disorders of pyruvic acid metabolism, 


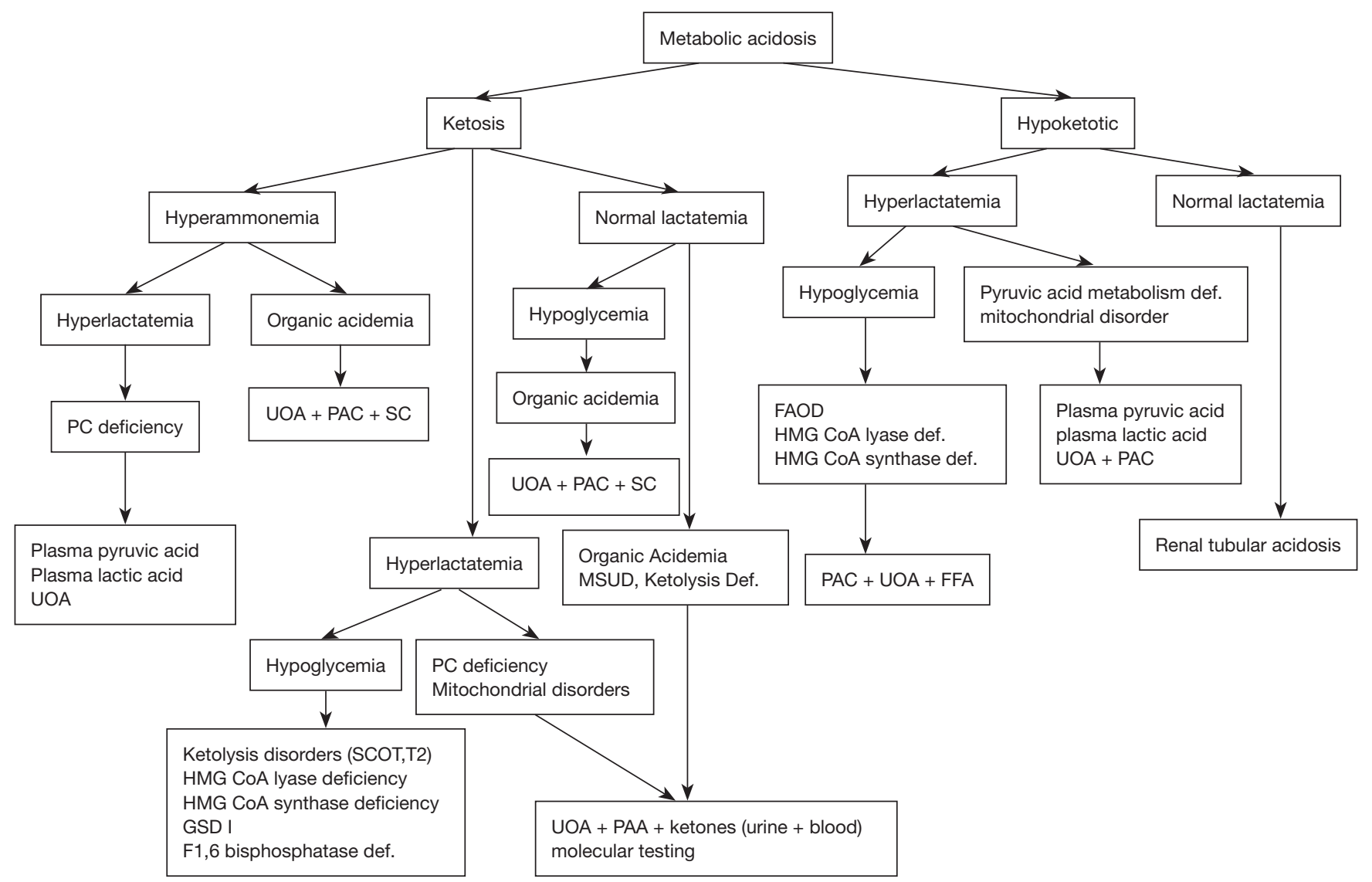

Figure 1 Metabolic Acidosis Workup Algorithm. PC, pyruvate carboxylase; UOA, urine organic acids; SC, serum carnitine; MSUD, maple syrup urinary disease; FOAD, fatty acid oxidation disorder; PAC, plasma acylcarnitines; FAA, free fatty acids; PAA, plasma amino acids; GDS 1, glycogen storage disorder type 1.

as well as in respiratory chain defects (Figure 1).

\section{Blood glucose}

Severe hypoglycemia is a life-threatening condition found in many metabolic disorders, including disorders of protein metabolism such as organic acidurias and certain amino acidopathies. However, critical hypoglycemia is a feature found in disorders directly affecting carbohydrate metabolism such as GSD, gluconeogenesis defects (glucose6-phosphatase deficiency, fructose-1,6-biphosphate deficiency), and mitochondrial fatty acid oxidation defects, which produce a severe depletion of circulating and reserve carbohydrates secondary to defective alternative energy production (Figure 2) $(13,14)$.

When evaluating hypoglycemia, a logical approach is to first consider whether the patient is ketotic or nonketotic. Disorders of mitochondrial fatty acid oxidation, carbohydrate metabolism, ketone body metabolism, and organic acidemias can all cause hypoglycemia. Disorders of mitochondrial fatty acid oxidation and ketogenesis including HMG-CoA lyase deficiency and HMG-CoA synthase deficiency as well as hyperinsulinemia are associated with hypoketotic hypoglycemia with or without severe metabolic acidosis, whereas other disorders such as organic acidemias, defect of ketone body metabolism and less commonly maple syrup urine disease (MSUD), typically cause ketotic hypoglycemia $(15,16)$.

During normal physiological conditions when hypoglycemia ensues, there is the simultaneous onset of hepatic glycogen-to-glucose conversion and an increase in the catabolism of free fatty acids. Mitochondrial fatty acid oxidation defects produce profound hypoglycemia due to the depletion of circulating glucose and hepatic glycogen 


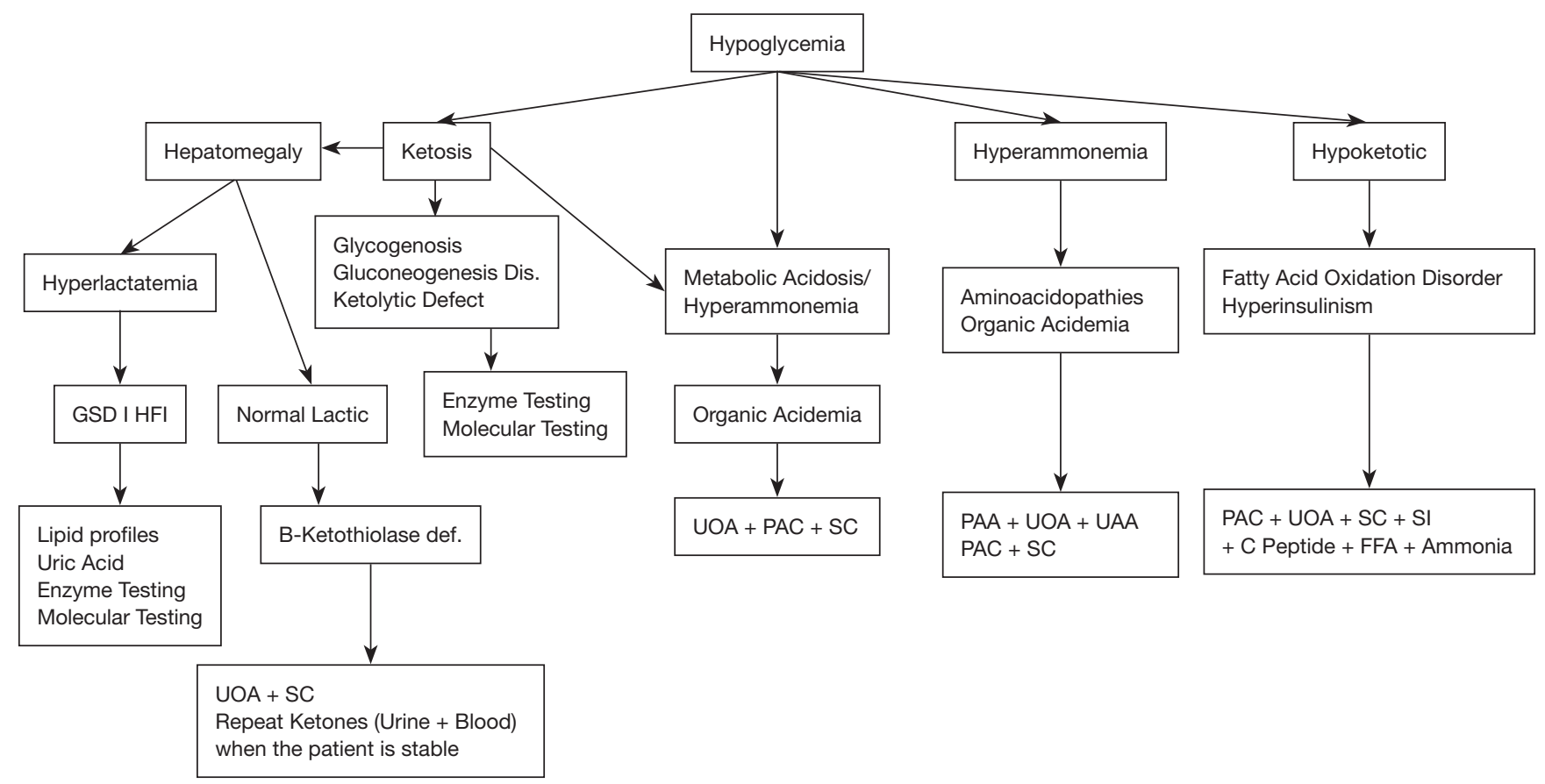

Figure 2 Hypoglycemia Workup Algorithm. GDS 1, glycogen storage disorder type 1; HFI, hereditary fructose intolerance; UOA, urine organic acids; SC, serum carnitine; PAC, plasma acylcarnitines; PAA, plasma amino acids; UAA, urine amino acids; SI, serum insulin; FAA, free fatty acids.

stores arising from the inability to metabolize fatty acids to meet energy requirements. In these defects, there is also the inability to rescue hypoglycemia and reduced production of acetyl-CoA due to decreased flux through the betaoxidation spiral, which affects ketone body production.

In GSD, there is impaired conversion of hepatic glycogen into circulating glucose during fasting, thus depleting available carbohydrates; the hypoglycemia is associated with hepatomegaly, mild to severe hepatic dysfunction, and hyperlactatemia. However, hypoglycemia might be absent in GSD type II (Pompe disease, or lysosomal acid maltase deficiency) as cytoplasmic glycogen metabolism is spared and glycogen accumulates only in lysosomes and in the early stages of GSD type IV. Hypoglycemia can also be found in disorders of carbohydrate metabolism like the galactosemias or hereditary fructose intolerance. In classic galactosemia, accumulated galactose-1-phosphate inhibits phosphoglucomutase to impair glycolysis, whereas in hereditary fructose intolerance, accumulated fructose-1-phosphate inhibits both gluconeogenesis and glycogenolysis (17-20).

A history of the time-based relationship of hypoglycemia to feeding can be helpful in making the diagnosis.
Hypoglycemia in the postprandial state or after a short duration fast ( $<4$ hours) is usually associated with a glucose overutilization problem, such as hyperinsulinism, whereas hypoglycemia following a prolonged fast ( $>8$ hours) is suggestive of a fatty acid oxidation defect. Hypoglycemia after a fast of intermediate duration (4-8 hours) may be due to a glycogenosis or a disorder affecting gluconeogenesis. Other basic laboratory findings may also be helpful; for example, hypoglycemia in the presence of hepatic fibrosis and cirrhosis might be the only finding in hereditary tyrosinemia type I.

\section{Ammonia}

Like hypoglycemia, hyperammonemia is also a lifethreatening condition; therefore, the plasma ammonia level should be tested in all patients with alteration of consciousness and encephalopathy, particularly young children. Hyperammonemia can be caused by many nonmetabolic conditions, including liver disease, portocaval shunt, glutamate dehydrogenase hyperactivity, or valproic acid toxicity. However, a marked elevation of ammonia, typically 10-100 times the upper limit of normal, can 


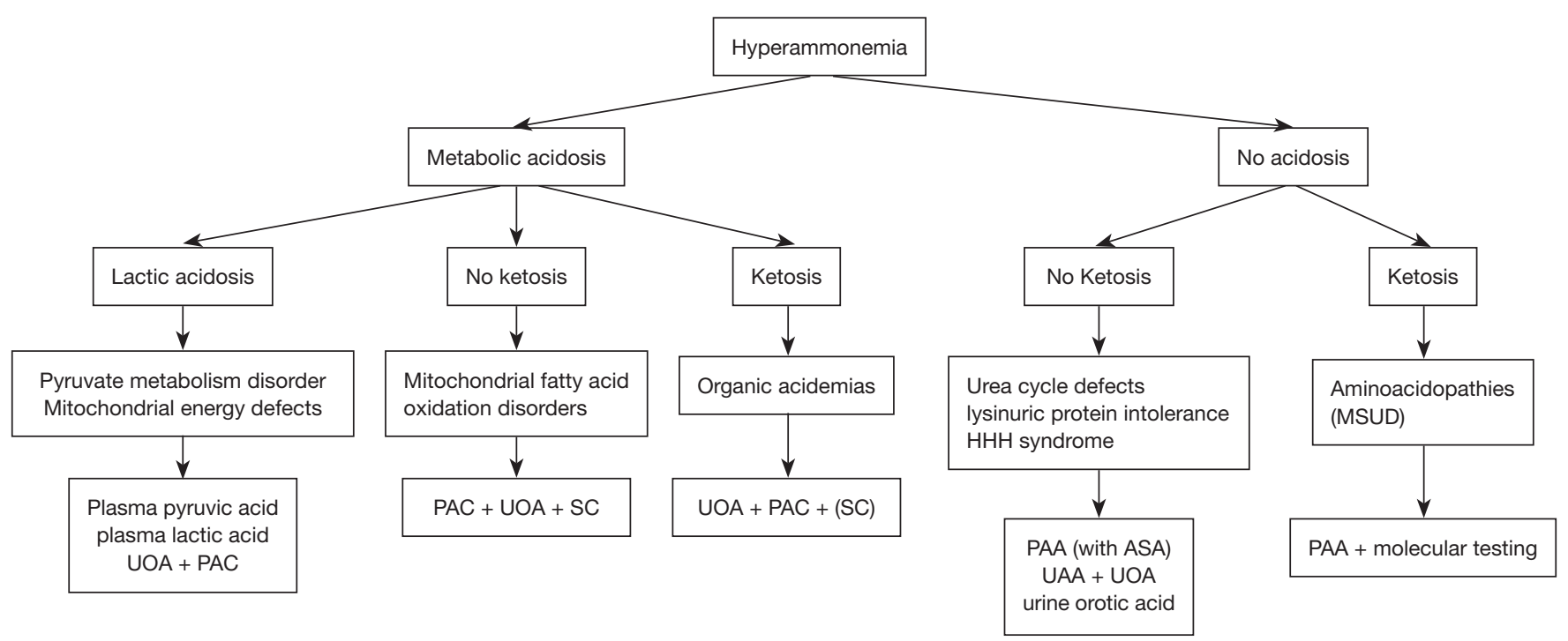

Figure 3 Hyperammonemia Workup Algorithm. UOA, urine organic acids; PAC, plasma acylcarnitines; SC, serum carnitine; PAA, plasma amino acids; ASA, argininosuccinic acid; UAA, urine amino acids.

be associated with urea cycle disorders. Although some organic acidemias and disorders of mitochondrial fatty acid oxidation may also cause hyperammonemia, it is usually less significant.

Ammonia, the neurotoxic byproduct of amino acid deamination, is converted to excretable urea by the urea cycle in a series of enzymatic steps occurring either in the cytosol or in the mitochondrion. Although very efficient under normal conditions, the urea cycle is a comparatively fragile metabolic process that can be affected by inherited metabolic disorders through a variety of different mechanisms (Figure 3).

Urea cycle disorders are inherited deficiencies in any of the enzymes of the urea cycle, or in the production of the allosteric cofactor $\mathrm{N}$-acetylglutamine, resulting in severe primary hyperammonemia. Hyperammonemia is also a relatively common secondary finding in organic acidurias, wherein accumulated substrates or organic acid intermediates inhibit the proximal urea cycle enzyme $\mathrm{N}$-acetylglutamate synthase (NAGS), producing an overall reduction of the urea cycle detoxifying efficacy. Among the organic acidurias, propionic acidemia and methylmalonic acidemia in particular can present with intermittent secondary hyperammonemia due to the inhibitory capability of accumulated propionyl-CoA. Metabolic derangements in which circulating levels of ornithine, citrulline, or arginine are decreased due to renal losses or reduced endogenous production can also produce hyperammonemia, since all three are urea cycle intermediates. When circulating or intracellular levels of these amino acids fall, the efficiency of the urea cycle can decrease, resulting in hyperammonemia; the most profound losses of amino acids important to the urea cycle are found in cystinuria and lysinuric protein intolerance where the renal reabsorption of ornithine and arginine can be severely reduced due to competition for a shared dibasic amino acid transporter. In contrast, mitochondrial fatty acid oxidation disorders can present with hyperammonemia due to the combined effects of substrate depletion and urea cycle inhibition by toxic acylcarnitine species. The level of acetyl-CoA, the end product of fatty acid beta oxidation, is decreased when overall flux through the pathway is reduced. Acetyl-CoA is required for the production of $\mathrm{N}$-acetylglutamate, which allosterically activates the enzyme carbamoyl phosphate synthetase 1 (CPS1); CPS1 converts ammonia to carbamoyl phosphate in the rate-limiting first step of the urea cycle. In certain long-chain fatty acid oxidation defects, fatty acylation of an active site residue of CPS1 directly affects the urea cycle detoxification capacity. Finally, some disorders can produce hyperammonemia secondary to organ damage. For example, in lysosomal disorders including the mucopolysaccharidoses as well as in some peroxisomal disorders, the accumulation and storage of complex large molecules within the liver leads to hepatocellular damage, which in turn causes a decrease in urea cycle efficiency (21-26). 


\section{Lactic acid}

The physiological balance of circulating lactic acid sustained by lactic acid production through cytoplasmic glycolysis and multi-tissue mitochondrial consumption can be disturbed by both non-metabolic and metabolic conditions. Lactic acidosis can occur due to an increase in lactate production, or a decrease in its metabolism. Most metabolic disorders presenting with hyperlactatemia have concurrent ketosis, with the exception of pyruvic dehydrogenase (PDH) deficiency, glycogenosis type-I or certain fatty acid oxidation disorders (27).

Lactic acidosis is most often caused by tissue hypoxia due to poor circulation or inadequate oxygen supply from multiple causes, including cardiogenic or hypovolemic shock. Non-metabolic sources of hyperlactatemia are not usually accompanied by ketosis. However, several IEMs including organic acidurias, disorders of mitochondrial energy metabolism, or defects of gluconeogenesis can also present with lactic acidosis. Once a non-metabolic etiology of hyperlactatemia has been ruled out, the most common origins of hyperlactatemia secondary to mitochondrial energy disruption by toxic metabolites are fatty acid oxidation disorders, organic acidurias, and in very rare cases, urea cycle defects. Other inherited causes of persistent hyperlactatemia include disorders of glycogen metabolism, disorders affecting gluconeogenesis, and disorders directly affecting the Krebs cycle or pyruvic acid metabolism. In gluconeogenesis defects such as fructose- 1,6 phosphatase deficiency and GSD type IA [glucose-6-phosphatase (G-6PD) deficiency], hyperlactatemia peaks when fasting or in hypoglycemic situations. In disorders affecting glycogen degradation, hyperlactatemia peaks postprandial. Hyperlactatemia can differ in disorders directly affecting pyruvic acid metabolism $(28,29)$. In pyruvate dehydrogenase (PDH) deficiency, alpha-ketoglutarate dehydrogenase deficiency, and respiratory chain disorders, hyperlactatemia usually occurs in the fed state, whereas in pyruvate carboxylase deficiency, hyperlactatemia occurs in both fasting and fed states. When evaluating a patient for hyperlactatemia, an often-overlooked relationship is the balance between the lactic acid/pyruvic acid and 3-OH butyric acid/acetoacetic acid ratios. The plasma lactic acid/pyruvic acid molar ratio correlates with the NAD+/ $\mathrm{NADH}$ ratio and can indirectly reflect the cytosolic redox state (30-32), while the 3-hydroxybutyric acid/acetoacetic acid ratio reflects the intramitochondrial redox state.

\section{Ketones}

The ketone bodies 3-hydroxybutyric acid, acetoacetic acid, and acetone are the natural end products of mitochondrial fatty acid beta-oxidation. Ketonuria, an increase in the urinary excretion of ketones, is found physiologically in late infancy, childhood and adolescence, but is not considered normal in the neonate. Physiological ketosis is not accompanied by metabolic acidosis, hyperlactatemia, or hypoglycemia, markers of metabolic stress; it is a common finding following fasting, vomiting, consumption of a ketogenic diet, or states of increased catabolism. However, because ketones are organic acids, severe ketonuria that produces metabolic acidosis should not be considered physiological, and indicate an IEM. The timing of ketonuria in relation to feeding or fasting is an important differentiator that can point to the potential type of underlying metabolic disorder. For instance, severe ketonuria with fasting hypoglycemia, or postprandial hyperglycemia with hyperlactatemia are common findings in certain disorders of glycogenosis. In contrast, pure marked ketonuria in fed and fasting states can occur in ketone body handling disorders such as succinyl-CoA transferase (SCOT) deficiency or beta-ketothiolase (BKT) deficiency. MSUD and organic acidemias like propionic acidemia and isovaleric acidemia can present with high anion gap metabolic acidosis and ketonuria. Unlike other markers of metabolic stress, ketosis is clinically relevant both when increased and when absent. While a severe reduction in the excretion of ketones along with low circulating glucose levels (hypoketotic hypoglycemia) is a common finding in vomiting, anorexia, or generalized catabolic states, this pattern is also a significant indicator of a potential disorder of mitochondrial fatty acid oxidation, with or without glucose overutilization. It is important to note, however, that several mitochondrial fatty acid oxidation disorders can present with intermittent episodes of mild to severe ketonuria when the affected enzyme is distal enough in the beta-oxidation degradative pathway that long-chain fatty acid metabolism is still capable of generating some ketones, as in the case of 3-hydroxyacylCoA dehydrogenase (HAD) deficiency or medium-chainacyl-CoA dehydrogenase (MCAD) deficiency, or in verylong-chain-acyl-CoA dehydrogenase (VLCAD) deficiency, where the oxidation of unsaturated fatty acids is primarily affected, while the oxidation of saturated fatty acids remains mostly intact (15,33-36). 


\section{Additional considerations}

Aside from those investigations listed above, other important initial tests include CBC, liver function tests, coagulation studies, creatine kinase levels, renal function tests, BUN, uric acid, lipid profiles and CSF cell study and CSF glucose. Non-laboratory tests can also be critical to the diagnosis of an IEM. Neuroimaging studies including MRI and MR spectroscopy may show specific brain findings characteristic of certain inherited defects, such as symmetrical basal ganglia and thalamic involvement seen in mitochondrial disorders, subdural effusions and reduced opercularization seen in glutaric acidemia type I (GA1), an absent creatine peak demonstrated by MR spectroscopy in congenital disorders of creatine metabolism, and white matter changes in disorders such as X-linked adrenoleukodystrophy and metachromatic leukodystrophy (37).

Based on the patient's clinical presentation and the outcome of routine laboratory analyses, once the suspicion of a metabolic disorder has been established, the most informative subsequent testing will depend on the potential disorder or group of disorders included in the differential diagnosis. Laboratory tests that cast a wide net and can be used to diagnose multiple IEMs include urine organic acid, plasma and urine amino acid, plasma acylcarnitine, and serum very long chain fatty acid analyses. Because secondary carnitine deficiency resulting from carnitine loss as acylcarnitine esters in the urine is a common finding in many metabolic disorders including fatty acid oxidation disorders and organic acidurias, serum carnitine quantification should also be included when evaluating a patient for a potential metabolic disorder. When such diagnostic tests are ordered, the most informative specimen type depends not only on the expected abnormal metabolites, but also whether the potential disorder is due to an enzyme deficiency or a transporter defect (Table 2) $(38,39)$.

Some disorders produce increases in circulating intermediate metabolites that, through their characteristic pattern, form the basis for diagnosis and dietary monitoring (i.e., increased plasma phenylalanine and decreased tyrosine in phenylketonuria, elevated free fatty acids in fatty acid oxidation disorders, or elevated branched-chain amino acids in MSUD); other disorders result in increased excretion of key metabolites which can be used for the same purposes (i.e., elevated urine cystine in cystinuria), while some disorders produce both. Elevations of abnormal metabolites in plasma can also be detected in urine if the blood concentration reaches the renal threshold for that
Table 2 Disorders by metabolic stress state

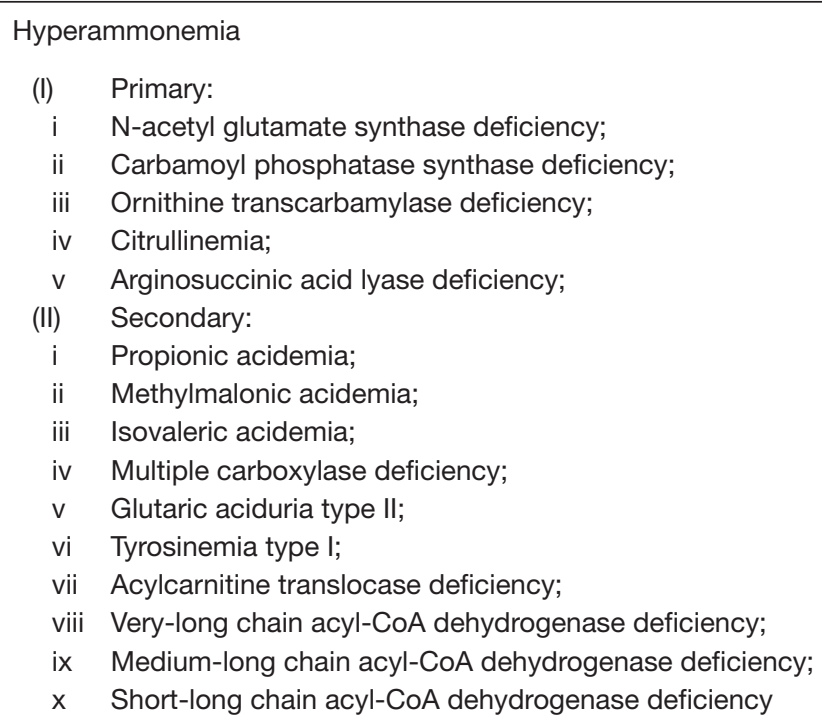

Metabolic acidosis

(I) Methylmalonic acidemia;

(II) Propionic acidemia;

(III) Isovaleric acidemia;

(IV) 3-hydroxy-3-methylglutaryl CoA lyase deficiency;

(V) Multiple carboxylase deficiency;

(VI) Pyruvate dehydrogenase complex deficiency;

(VII) Pyruvate carboxylase complex deficiency;

(VIII) Maple syrup urinary disease;

(IX) Glutaric aciduria type I;

(X) Glutaric aciduria type II;

(XI) Short-long chain acyl-CoA dehydrogenase deficiency;

(XII) Long chain 3-OH acyl-CoA dehydrogenase deficiency;

(XIII) B-Ketothiolase deficiency;

(XIV) Acetoacetate CoA ligase deficiency;

(XV) Mitochondrial respiratory/electron chain defects;

(XVI) Galactosemia;

(XVII) Glycogen storage disorder type I;

(XVIII) Fructose-1,6 diphosphatase deficiency;

(XIX) Hereditary fructose intolerance;

(XX) Tyrosinemia type I

Ketonuria

(I) Propionic acidemia;

(II) Methylmalonic acidemia;

(III) Isovaleric acidemia;

(IV) Multiple carboxylase deficiency;

(V) Glutaric aciduria type II;

(VI) Maple syrup urinary disease;

(VII) B-Ketothiolase deficiency;

(VIII) Acetoacetate CoA ligase deficiency;

(IX) Pyruvate carboxylase complex deficiency;

(X) Fructose-1,6 diphosphatase deficiency;

(XI) Glycogen storage disorder, type I

Table 2 (continued) 
Table 2 (continued)

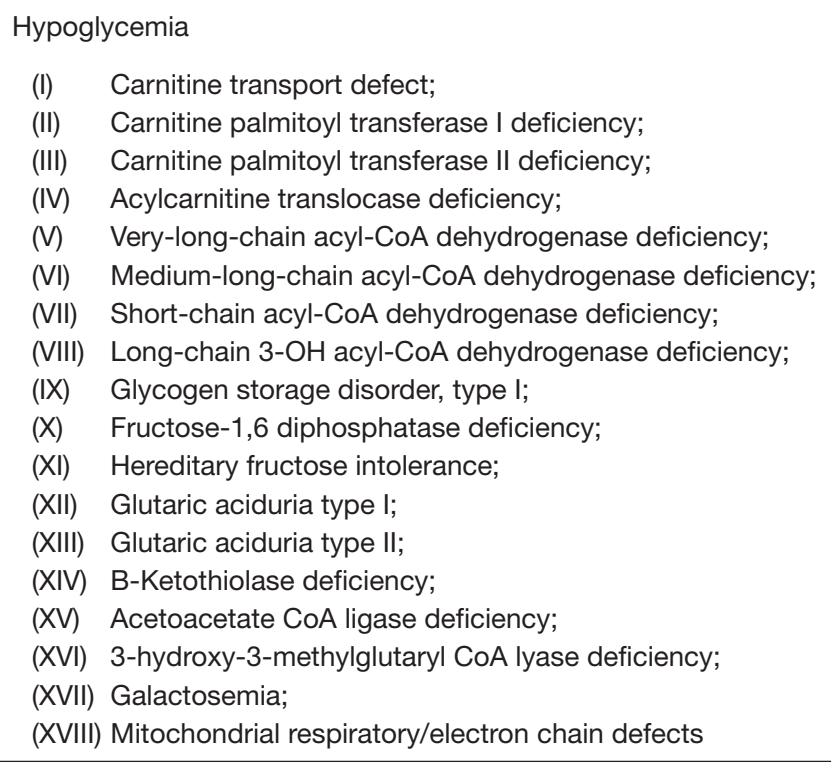

While most disorders have a predominant metabolic stress state based on the pathophysiology of the metabolic derangement, many disorders may present with multiple stress states due to interactions with other metabolic pathways.

analyte. In general, disorders of amino acid metabolism that affect proximal metabolic steps (i.e., phenylketonuria or MSUD) will lead to abnormal plasma amino acid profiles. Disorders of amino acid metabolism which impact distal enzymatic steps (i.e., isovaleric acidemia or glutaric aciduria type I) may or may not show abnormalities on plasma amino acids analysis, but typically yield abnormal urine organic acid profiles. Disorders that affect amino acid transporters in the kidney (i.e., cystinuria or lysinuric protein intolerance) are usually best diagnosed by a urine amino acid profile. Conditions affecting different steps in a shared metabolic pathway may produce abnormal patterns of common intermediate metabolites; in such cases, further testing may be required to narrow down the diagnosis, as when an elevated level of 3-hydroxyisovaleryl/2-methyl-3-hydroxybutyrylcarnitine $(\mathrm{C} 5 \mathrm{OH})$ is detected in a plasma acylcarnitine profile. This analyte is found in several disorders affecting branched-chain amino acid metabolism. In such cases, urine organic acid analysis or further complementary biochemical or molecular tests may be required to confirm the initial findings, or to make a definitive diagnosis (Figures 1-3).

In some other conditions, enzyme activity analysis and/or molecular analysis may be necessary for a specific diagnosis; it should be noted that some enzyme tests require invasive procedures such as a liver biopsy for hepatic glycogen storage disease or skin biopsy for fibroblast culture for fatty acid oxidation studies (28,40-42). In cases where a patient finally, in spite of the reduction in morbidity and mortality following the implementation of widespread NBS, improvements in therapy, and the identification of lateronset clinical presentation of most IEM, it is inevitable that some patients will pass away before a diagnosis is made. In these instances, it is important to collect post mortem blood and urine specimens whenever possible, to aid in identifying any underlying metabolic disorder (43).

\section{Defects of organelle metabolism}

Patients with metabolic defects that affect an entire organelle usually present with slowly progressive encephalopathies and systemic symptoms. Basic laboratory investigations such as a CBC, liver and renal function tests, ferritin, and bone $\mathrm{X}$-rays, among others, can provide information about the baseline health status, convey evidence of possible coexisting diseases, and narrow the differential diagnosis. For example, pancytopenia and hyperferritinemia suggest Gaucher disease. Severe liver disease is seen in Niemann-Pick C (NPC). Neurological deficits can result in undernutrition, iron deficiency, anemia, and low albumin. Low levels of high-density lipoprotein (HDL) are occasionally observed in untreated Gaucher disease and NPC. Skeletal surveys are used to detect dysostosis multiplex or cartilage stippling, seen in many lysosomal storage diseases (LSD) and peroxisomal biogenesis disorders (44-46). Pathology studies including histology, histopathology, immunochemistry and electron microscopy (EM) can provide some diagnostic clues as well: for example, cytoplasmic vacuoles detected in lymphocytes or macrophages are suggestive of LSD (44).

\section{Biomarkers}

For some metabolic disorders, substances in different body fluids can be used as indicators of both the presence and the progression of the disease, as well as for monitoring the effect of therapy. For example, creatine kinase (CK or CPK) is a non-specific indicator of metabolic myopathy as is found in mitochondrial fatty acid oxidation defects, glycogen storage diseases, and Pompe disease. Circulating cytokines FGF-2 1 and GDF-15 have been recently identified as potential biomarkers of mitochondrial myopathy (47). 
Using micro-RNAs (miRNA) nucleotides as biomarkers for mitochondrial disorders has also been proposed. MicroRNAs regulate gene expression with high specificity on the post-transcriptional level, and distinctive patterns of micro-RNAs are associated with various disorders. There is evidence demonstrating that micro-RNA $9 / 9^{*}$ pattern is associated with mitochondrial encephalopathy, lactic acidosis and stroke-like episodes (MELAS) or the myoclonic epilepsy with ragged-red fibers (MERRF) phenotype (47). Plasma chitotriosidase (CHITO) analysis is commonly used as the first tier screen in the diagnosis of Gaucher disease, sphingomyelinase deficiency (Niemann-Pick disease type $\mathrm{A} / \mathrm{B}$ ) and NPC. A marked elevation of CHITO is seen in untreated Gaucher disease and a moderate elevation is found in NPA, B and C (48). Lack of specificity is one of the problems encountered when routine laboratory tests are abnormal. Physicians should keep in mind that other common conditions such as inflammation or infection can also cause elevations of certain biomarkers. Therefore, it is imperative that all laboratory results be interpreted in conjunction with the patient's clinical findings (Table 3).

\section{Future directions}

Since the first inborn error of metabolism was described in 1908, information on pathophysiology, etiology, prevalence, classification, diagnosis and treatment has expanded tremendously. New metabolic disorders are being described on a regular basis, and as our understanding of disease mechanisms continues to grow, additional disorders are likely to be discovered, including those due to defects in metabolic regulatory processes. NBS will continue to expand due to advancements in high throughput technology and the development of new therapies for additional metabolic conditions. With the availability of specific treatment for some LSD conditions, these disorders are already included in the NBS panels for several states, and inclusion of LSD in NBS will likely increase as therapy for additional disorders is developed. Direct enzyme activity assay from a dried-blood spot (DBS) was first developed more than a decade ago by Chamoles and team. Since then, interest in LSD NBS has grown considerably. Currently, the activity of multiple lysosomal enzymes in DBS can be measured simultaneously by digital microfluidics or multiplex-MS/MS (49). At present, 7 LSDs can be ascertained by NBS in DBS: Pompe, Krabbe, Gaucher, Niemann Pick A/B, Mucopolysaccharidosis I, and Fabry disease. Some of these disorders have already been included in expanded NBS panels in several US states and in some other countries (2).

Metabolomics is a new approach to the diagnosis of IEM, especially when the clinical presentations are nonspecific. Metabolomic analysis is focused on the complete set of all small molecule metabolites present in biological specimens. MS and nuclear magnetic resonance (NMR) are the main analytical techniques, although ion mobility spectrometry (IMS) combined with high resolution MS provides better coverage (50). This approach is sometimes called untargeted analysis due to the larger scope of detection versus traditional targeted assays for measuring amino acids, acylcarnitines, organic acids, purines, pyrimidines, and carnitine biosynthesis intermediates. It has been estimated that more than 700 different metabolites linked to IEM and more than 400 endogenous metabolites identified by untargeted MS-based metabolomics platforms cannot be detected by a combination of all targeted assays $(50,51)$. Aside from aiding diagnosis, metabolomics also can lead to the discovery of new IEM, novel biomarkers, and a better overall understanding of IEM and the regulation of metabolic processes (51). The inherent challenges associated with the large amount of data generated by high-throughput technologies include how to track, store, and share the data. It is crucial to develop advanced bioinformatics solutions, comprehensive openaccess databases, and computer methodologies and software to convert the huge amount of data generated by this approach into effective clinically actionable tools that can aid decision-making.

\section{Conclusions}

The field of IEM continues to grow as evolving technologies like NGS and comprehensive metabolomic profiling strategies provide deeper insight into mechanisms of disease and phenotypic differences between individuals with the same disorder. It has become increasingly apparent that the classical forms of IEM, with presentation in infancy or childhood, are just the tip of the iceberg. Milder variants that may not cause clinical symptoms until adulthood or in states of increased metabolic stress (pregnancy, infections, surgery, ketogenic diet), resulting in a pool of patients likely to be missed by NBS $(52,53)$. Inherited metabolic diseases may often be overlooked in the differential diagnosis for older patients or children who had a normal newborn screen, but case reports suggest that atypical presentations may be relatively common in this patient population (54). 
Table 3 Biochemical genetics tests that should be ordered depending on the suspected IEM

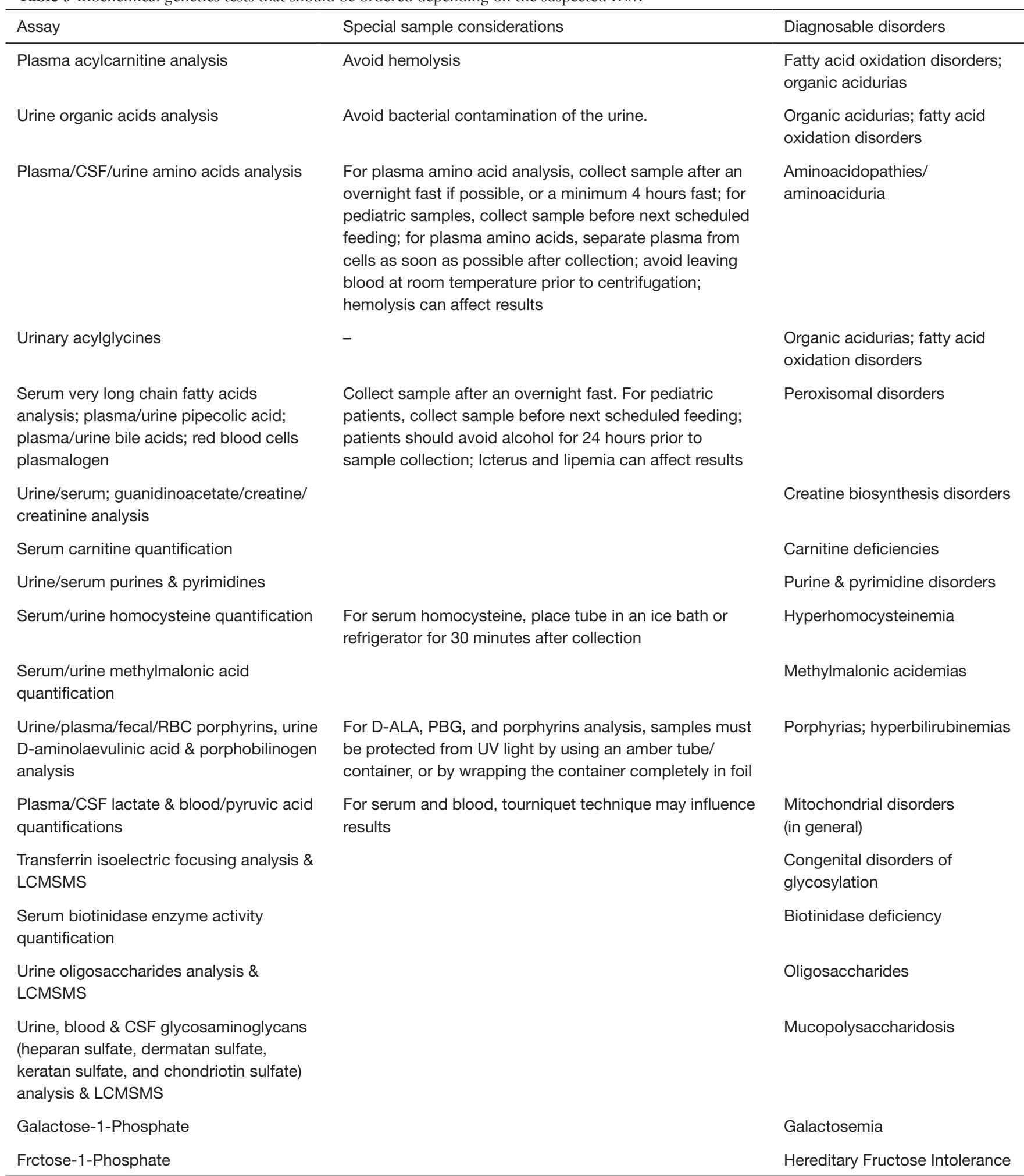

Table 3 (continued) 
Table 3 (continued)

\begin{tabular}{|c|c|c|}
\hline Assay & Special sample considerations & Diagnosable disorders \\
\hline $\begin{array}{l}\text { Plasma chitotriosidase (CHITO); } \\
\text { plasma oxysterols; urine sulfatides; } \\
\text { plasma creatine kinase (CK), aspartate } \\
\text { aminotransferase (AST) and alanine } \\
\text { aminotransferase (ALT), lactate } \\
\text { dehydrogenase (LDH), \& urine hexose } \\
\text { tetrasaccharide (HEX4 or glucose } \\
\text { tetrasaccharide, Glc4) }\end{array}$ & & $\begin{array}{l}\text { Niemann Pick A/B/C; Niemann } \\
\text { Pick C; metachromatic } \\
\text { leukodystrophy (MLD); } \\
\text { Pompe disease }\end{array}$ \\
\hline
\end{tabular}

In all groups of disorders, there are individual conditions for which specific mass spectrometry-based analysis, specific enzyme analysis and/or molecular genetics analysis might be required to accurately make a diagnosis. CSF, cerebrospinal fluid; RBC, red blood cell; LCMSMS, liquid chromatography tandem mass-spectrometry.

Although the sheer number of IEM is daunting, a systematic and logical approach to test selection in a patient with a clinical presentation of a metabolic defect, regardless of age, can lead to a high degree of diagnostic success.

\section{Acknowledgements}

Dr. Bonilla Guerrero and Dr. Salazar would like to thank Dr. Charles M. Strom for his mentorship, guidance, and leadership over the years.

\section{Footnote}

Conflicts of Interest: The authors have no conflicts of interest to declare.

\section{References}

1. Gilbert-Barness E, Farrell PM. Approach to diagnosis of metabolic diseases. Transl Sci Rare Dis 2016;1:3-22.

2. Recommended Uniform Screening Panel. Available online: https://www.hrsa.gov/advisory-committees/heritabledisorders/rusp/index.html

3. Clark JF, Cecil KM. Diagnostic methods and recommendations for the cerebral creatine deficiency syndromes. Pediatr Res 2015;77:398-405.

4. Bonilla Guerrero R, Karen K, Denise S, et al. The Porphyrias. In: Sarafloglou K. Editor. Pediatric Endocrinology and Inborn Errors of Metabolism. 2nd edition. New York: McGraw Hill, 2017.

5. Jaeken J, Peanne R. What is new in CDG? J Inherit Metab Dis 2017;40:569-86.

6. Korman SH. Inborn errors of isoleucine degradation: a review. Mol Genet Metab 2006;89:289-99.

7. Calvin J, Hogg S. Biochemical investigation for inborn errors of metabolism in adults presenting with neurological disorders. Adv Clin Neurosci Rehabil 2010;9:12-6.

8. Sharer JD. An overview of biochemical genetics. Curr Protoc Hum Genet 2016;89:17.1.1-17.1.16.

9. Wanders RJ. Metabolic and molecular basis of peroxisomal disorders: a review. Am J Med Genet A 2004;126A:355-75.

10. Wraith JE. Lysosomal disorders. Semin Neonatol 2002;7:75-83.

11. Vaidyanathan K, Narayanan MP, Vasudevan DM. Organic acidurias: an updated review. Indian J Clin Biochem 2011;26:319-25.

12. Kompare M, Rizzo WB. Mitochondrial fattyacid oxidation disorders. Semin Pediatr Neurol 2008;15:140-9. 
13. Vockley J, Singh RH, Whiteman DA. Diagnosis and management of defects of mitochondrial beta-oxidation. Curr Opin Clin Nutr Metab Care 2002;5:601-9.

14. Douillard C, Mention K, Dobbelaere D, et al. Hypoglycaemia related to inherited metabolic diseases in adults. Orphanet J Rare Dis 2012;7:26.

15. Bennett MJ, Weinberger MJ, Kobori JA, et al. Mitochondrial short-chain L-3-hydroxyacyl-coenzyme A dehydrogenase deficiency: a new defect of fatty acid oxidation. Pediatr Res 1996;39:185-8.

16. Ogier de Baulny H. Management and emergency treatments of neonates with a suspicion of inborn errors of metabolism. Semin Neonatol 2002;7:17-26.

17. Kilimann MW, Oldfors A. Glycogen pathways in disease: new developments in a classical field of medical genetics. J Inherit Metab Dis 2015;38:483-7.

18. Heese BA. Current strategies in the management of lysosomal storage diseases. Semin Pediatr Neurol 2008;15:119-26.

19. Leslie ND. Insights into the pathogenesis of galactosemia. Annu Rev Nutr 2003;23:59-80.

20. Li H, Byers HM, Diaz-Kuan A, et al. Acute liver failure in neonates with undiagnosed hereditary fructose intolerance due to exposure from widely available infant formulas. Mol Genet Metab 2018;123:428-32.

21. Singh RH, Rhead WJ, Smith W, et al. Nutritional management of urea cycle disorders. Crit Care Clin 2005;21:S27-35.

22. Ogier de Baulny H, Schiff M, Dionisi-Vici C. Lysinuric protein intolerance (LPI): A multi organ disease by far more complex than a classic urea cycle disorder. Mol Genet Metab 2012;106:12-7.

23. Walker V. Ammonia toxicity and its prevention in inherited defects of the urea cycle. Diabetes Obes Metab 2009; 11:823-35.

24. Iyer H, Sen M, Prasad C, et al. Coma, hyperammonemia, metabolic acidosis, and mutation: lessons learned in the acute management of late onset urea cycle disorders. Hemodial Int 2012;16:95-100.

25. Häberle J. Clinical and biochemical aspects of primary and secondary hyperammonemic disorders. Arch Biochem Biophys 2013;536:101-8.

26. Auron A, Brophy PD. Hyperammonemia in review: pathophysiology, diagnosis, and treatment. Pediatr Nephrol 2012;27:207-22.

27. Gutierrez G, Williams JD. The riddle of hyperlactatemia. Crit Care 2009;13:176.

28. Pasquali M, Monsen G, Richardson L, et al.
Biochemical findings in common inborn errors of metabolism. Am J Med Genet C Semin Med Genet 2006;142C:64-76.

29. Saudubray JM, Rabier D. Biomarkers identified in inborn errors for lysine, arginine, and ornithine. J Nutr 2007;137:1669S-72S.

30. Debray FG, Mitchell GA, Allard P, et al. Diagnostic accuracy of blood lactate-to-pyruvate molar ratio in the differential diagnosis of congenital lactic acidosis. Clin Chem 2007;53:916-21.

31. Haas RH, Parikh S, Falk MJ, et al. The in-depth evaluation of suspected mitochondrial disease. Mol Genet Metab 2008;94:16-37.

32. Sass JO, Fukao T, Mitchell GA. Inborn Errors of Ketone Body Metabolism and Transport: An Update for the Clinic and for Clinical Laboratories. J Inborn Erros of Metabolism 2018;6:1-7.

33. Wüst RC, Visser G, Wanders RJ, et al. Ketones and inborn errors of metabolism: old friends revisited. J Inherit Metab Dis 2017;40:3-4.

34. Ogier de Baulny H, Saudubray JM. Branched-chain organic acidurias. Semin Neonatol 2002;7:65-74.

35. Wraige E, Champion MP, Turner C, et al. Fat oxidation defect presenting with overwhelming ketonuria. Arch Dis Child 2002;87:428-9; discussion 428-9.

36. Patel JS, Leonard JV. Ketonuria and medium-chain acylCoA dehydrogenase deficiency. J Inherit Metab Dis 1995;18:98-9.

37. Garcia-Cazorla A WN, Hoffman GF. Neurological disease. In: Hoffman GF ZJ, Nyhan WL. Editor. Inherited Metabolic Diseases: A clinical approach 2010;127-59.

38. Longo N, Amat di San Filippo C, Pasquali M. Disorders of carnitine transport and the carnitine cycle. Am J Med Genet C Semin Med Genet 2006;142C:77-85.

39. Zammit VA, Ramsay RR, Bonomini M, et al. Carnitine, mitochondrial function and therapy. Adv Drug Deliv Rev 2009;61:1353-62.

40. Vockley J, Andersson HC, Antshel KM, et al. Phenylalanine hydroxylase deficiency: diagnosis and management guideline. Genet Med 2014;16:188-200.

41. Frazier DM, Allgeier C, Homer C, et al. Nutrition management guideline for maple syrup urine disease: an evidence- and consensus-based approach. Mol Genet Metab 2014;112:210-7.

42. Camargo SM, Bockenhauer D, Kleta R. Aminoacidurias: Clinical and molecular aspects. Kidney Int 2008;73:918-25. 
43. Saudubray JM, Garcia-Cazorla A. Inborn Errors of Metabolism Overview: Pathophysiology, Manifestations, Evaluation, and Management. Pediatr Clin North Am 2018;65:179-208.

44. Tanpaiboon P, Lopez G. Lysosomal storage disorders and epilepsy. In: Pearl PL. Editor. Inherited Metabolic Epilepsies. 2nd edition. New York: Demos Medical Publishing, LLC, 2017.

45. Johnson BA, Dajnoki A, Bodamer O. Diagnosis of lysosomal storage disorders: Gaucher disease. Curr Protoc Hum Genet 2014;82:17.15.1-6.

46. Winchester B. Lysosomal diseases: diagnostic update. J Inherit Metab Dis 2014;37:599-608.

47. Finsterer J, Zarrouk-Mahjoub S. Biomarkers for Detecting Mitochondrial Disorders. J Clin Med 2018;7.

48. Ries M, Schaefer E, Luhrs T, et al. Critical assessment of chitotriosidase analysis in the rational laboratory diagnosis of children with Gaucher disease and NiemannPick disease type A/B and C. J Inherit Metab Dis 2006;29:647-52.

49. Elliott S, Buroker N, Cournoyer JJ, et al. Pilot study of newborn screening for six lysosomal storage diseases

Cite this article as: Bonilla Guerrero R, Salazar D, Tanpaiboon P. Laboratory diagnostic approaches in metabolic disorders. Ann Transl Med 2018;6(24):470. doi: 10.21037/atm.2018.11.05 using Tandem Mass Spectrometry. Mol Genet Metab 2016;118:304-9.

50. Tebani A, Afonso C, Marret S, et al. Omics-Based Strategies in Precision Medicine: Toward a Paradigm Shift in Inborn Errors of Metabolism Investigations. Int J Mol Sci 2016;17.

51. Miller MJ, Kennedy AD, Eckhart AD, et al. Untargeted metabolomic analysis for the clinical screening of inborn errors of metabolism. J Inherit Metab Dis 2015;38:1029-39.

52. Edmondson AC, Salant J, Ierardi-Curto LA, et al. Missed Newborn Screening Case of Carnitine Palmitoyltransferase-II Deficiency. JIMD Rep 2017;33:93-7.

53. Ganetzky RD, Bedoukian E, Deardorff MA, et al. Argininosuccinic Acid Lyase Deficiency Missed by Newborn Screen. JIMD Rep 2017;34:43-7.

54. Staels W, D'Haese J, Sercu E, et al. Medium-chain AcylCoA dehydrogenase deficiency presenting with neonatal pulmonary haemorrhage. Matern Health Neonatol Perinatol 2015;1:8. 